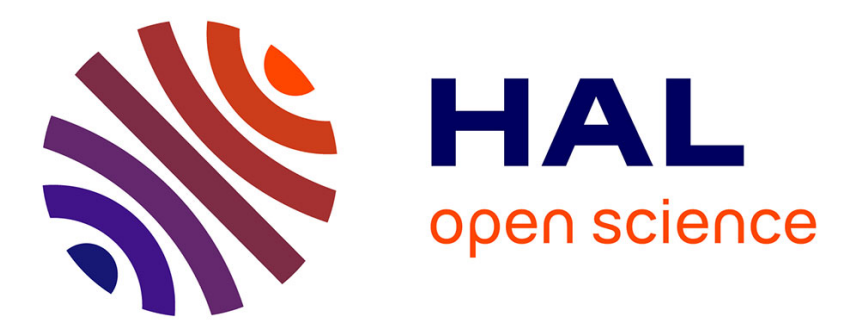

\title{
Deux dimensions du beau en géomorphologie. Essai sur le critère esthétique dans les sciences du relief
}

Christian Giusti

\section{To cite this version:}

Christian Giusti. Deux dimensions du beau en géomorphologie. Essai sur le critère esthétique dans les sciences du relief. L'Information géographique, 2014, 2014-3, pp.ISSN : 0020-0093. hal-01076213

\section{HAL Id: hal-01076213 \\ https://hal.science/hal-01076213}

Submitted on 21 Oct 2014

HAL is a multi-disciplinary open access archive for the deposit and dissemination of scientific research documents, whether they are published or not. The documents may come from teaching and research institutions in France or abroad, or from public or private research centers.
L'archive ouverte pluridisciplinaire HAL, est destinée au dépôt et à la diffusion de documents scientifiques de niveau recherche, publiés ou non, émanant des établissements d'enseignement et de recherche français ou étrangers, des laboratoires publics ou privés.

\section{(ㅇ)(1) $\$$}

Distributed under a Creative Commons Attribution - NonCommercial - NoDerivatives $\mid 4.0$ 


\title{
Titre
}

Deux dimensions du beau en géomorphologie. Essai sur le critère esthétique dans les sciences du relief.

\section{Title}

Two dimensions of beauty in geomorphology. Essay on the aesthetic criterion in landform sciences.

\section{Résumé}

Le prologue de cet essai esquisse les aspects contemporains de la géomorphologie. La première partie rappelle les deux sens du mot relief en français. L'article propose ensuite de porter un nouveau regard sur les formes et les processus du relief, d'une part en proposant une analyse esthétique de la toposphère, d'autre part en questionnant le statut socio-économique des composantes géomorphologiques des paysages de l'Anthropocène. L'épilogue est une invitation à fusionner jaillissement des émotion et éléments de compréhension.

\begin{abstract}
The prologue of this essay outlines the contemporary aspects of geomorphology. The first part recalls the two meanings of the word 'relief' in French. Then the paper proposes to take a fresh look on landforms and processes in geomorphology, on the one hand by deciphering the aesthetic dimension of the toposphere, on the other hand through the examination of the socio-economic status of geomorphic components of landscapes in the Anthropocene. The epilogue is an invitation to merge the outburst of emotions and the elements of understanding.
\end{abstract}

\section{Mots clé en français et en anglais}

Géographie, Géologie, Géomorphologie, Toposphère, Environnement, Patrimoine. Geography, Geology, Geomorphology, Toposphere, Environment, Heritage.

\section{Emplacement des figures (voir corps de l'article)}

5 figures (photos couleurs), 2 tableaux (dans le texte)

\section{Auteur et affiliation}

Christian GIUSTI

UFR de Géographie-Aménagement Paris-Sorbonne, UMR 8185 ENeC

Christian.Giusti@paris-sorbonne.fr 


\section{Deux dimensions du beau en géomorphologie.}

Essai sur le critère esthétique dans les sciences du relief.

\section{Prologue}

Le Mont Saint Helens ${ }^{1}$, ou Lawetlat'la pour les Cowlitz et Loowit pour les Klickitat, est un strato-volcan actif dont les formes, pures et symétriques en 1890, lui ont valu d'être surnommé le « Mont Fuji ${ }^{2}$ des Amériques ». Ce volcan situé à $150 \mathrm{~km}$ au sud de Seattle (Washington) et à $80 \mathrm{~km}$ au nord-est de Portland (Oregon), appartient à la chaîne et à l'arc volcanique des Cascades, partie intégrante de la « Ceinture de Feu » du Pacifique. Le 18 mai 1980, l'explosion catastrophique du Mont Saint Helens provoquait des dégâts considérables, entraînant dans la mort plus de 50 personnes. Un glissement de terrain déplaçant un volume de $2,3 \mathrm{~km}^{3}$ de matières diverses transformait le sommet sub-conique en un cratère échancré d'une largeur de 1,5 kilomètres, l'altitude sommitale du volcan passant de 2955 à 2549 mètres, soit une réduction de $406 \mathrm{~m}$ en terme de Reliefenergie, l'énergie du relief. «Les volcans sont peut-être, avec leur symétrie et leurs légères pentes paraboliques, les objets les plus étonnant d'un monde où dominent les topographies irrégulières. Si les volcans surplombent le paysage environnant, comme c'est le cas au nord-ouest des États-Unis, l'effet en est d'autant plus saisissant... Mais il est une autre beauté, plus profonde peut-être, qui réside dans la compréhension des phénomènes » (Gould, 1994).

À ma connaissance, il n'existe pas dans l'ordre de la géodiversité d'équivalent à On Growth and Form, le génial essai de D'Arcy Wentworth Thompson $(1917,1942)^{3}$, qui pensait que les biologistes de son époque avaient tendance à sous-estimer le poids des contraintes que les lois physiques et mécaniques imposent au développement des êtres vivants, pour privilégier le rôle des facteurs évolutifs comme déterminant principal de la forme et de la structure interne des organismes vivants (Gould, 2002, 1181-12084. Cohen, 2004). À l'opposé, l'une des erreurs les plus fantastiques jamais introduites en géomorphologie ${ }^{5}$ est due à William Morris Davis qui, dans l'article où il expose le modèle du geographical cycle, mal traduit en français par « cycle d'érosion », a voulu calquer le développement des formes du relief sur la

\footnotetext{
${ }^{1}$ http://en.wikipedia.org/wiki/Mount_St. Helens [consulté le 7 juin 2014]

${ }^{2}$ Voir l'article Mt Fuji: the beauty of a symmetric stratovolcano (Oguchi et Oguchi, in Migon, 2010).

${ }^{3}$ Traduction de Dominique Teyssié (D’Arcy Thompson, 1994), sur l'édition établie par J.T. Bonner en 1961.

${ }^{4}$ Dans la traduction de Marcel Blanc (Gould, 2006), il s'agit des pages 1652 à 1689 du chapitre 11.

${ }^{5}$ « Penck's conception that slope profiles are convex, plane, or concave, according to the circumstances of the uplifting action, is in my judgment one of the most fantastic errors ever introduced into geomorphology » (Johnson, 1940, 231, souligné par nous. Voir Giusti, 2012b).
} 
succession des âges de la vie, recourant à la métaphore vitaliste et organiciste analysée par V. Berdoulay (1982) : "The larva, the pupa and the imago of an insect, or the acorn, the fullgrown oak, and the fallen old trunk, are no more naturally associated as representing the different phases in the life history of a single organic species than are the young mountain block, the maturely carved mountain peaks and valleys, and the old mountain peneplain, as representing the different stages in the life history of a single geographical group » (Davis, 1899). Bien que la part de la pédagogie - Davis, écrit Baulig (1950), était « profondément, passionnément, on dirait presque agressivement pédagogue »-doive ici être faite, le devenir des formes du relief n'est pas comparable à la vie des êtres organiques pris comme individu ou comme espèce, car un relief n'est pas un être capable de se reproduire, ni un système de formes une population au sens biologique du terme. Même si la frontière séparant le biotique de l'abiotique se révèle bien moins étanche que ce que qui était admis (par ex., Butler, 1995. Stallins, 2006), il reste que, à l'instar des planètes, des étoiles, ou des galaxies, les formes du relief sont surtout des systèmes abiotiques qui, au fil du temps, ne cessent de recevoir, échanger et dissiper de l'énergie de façon complexe (Gell-Mann, 1994. Huggett, 2007). Ces systèmes ne sont pas toujours en évolution ou en transformation à travers les âges, car il arrive que des ensembles de formes puissent se maintenir plus ou moins longtemps de façon stationnaire en situation d'équilibre auto-entretenu (Hack, 1960. Klein, 1960).

Les reliefs de la Terre - océaniques, subaériens, ou sous-marins (plateformes continentales affectées par les marées et les diverses variations eustatiques : glaciaires, diastrophiques...) sont sous l'influence de forces internes et de mécanismes externes dont tous les paramètres ne cessent de s'ajuster pour produire des combinaisons plus ou moins stables, plus ou moins durables. Toute morphogenèse se produit en fonction d'arrangements variables et changeants impliquant à des degrés divers des processus tectoniques, eustatiques, climatiques, biologiques, anthropiques. Les lois générales de l'érosion chimique et/ou mécanique, des types d'écoulement et de transport (fluviaux, glaciaires, éoliens, diffus, concentré...), l'action des vagues, des courants marins, des forces de marée, la cinématique des plaques, le rôle des variations climatiques, celui des fluctuations du niveau des mers et de l'océan, les transformations des biomes, des sols, mais aussi l'accroissement de la population humaine, relèvent de cadres généraux, donc d'une approche nomothétique. Mais parce que chaque objet, quel que soit sa dimension, possède sa spécificité (le Bray n'est pas le Weald, le Pacifique n'est pas l'Atlantique, le Mont Saint Helens n'est pas le Mont Fuji), et aussi parce que les séquences d'événements sont uniques, contingentes et irréversibles, " ultimately, the 
question of questions boils down to the placement of the boundary between predictability under invariant law and the multifarious possibilities of historical contingency » (Gould, 1989, 290) ${ }^{6}$. Dans la métaphore du «perfect landscape », J.D. Phillips (2007) a poussé le curseur loin vers le pôle de la contingence : «Landforms and landscapes are circumstantial, contingent results of deterministic laws operating in a specific environmental context, such that multiple outcomes are possible $»^{7}$. Mais les formes et les modelés du relief relèvent tant des lois physico-chimiques (Anderson et Anderson, 2010) que de la contingence historique (Giusti, 2012a, 2012b), et l'on rappellera que, dès 1962, l'un des critiques les plus radicaux des vues davisiennes avait revendiqué dans son essai sur la théorie générale des systèmes et la géomorphologie la pertinence des idées développées par D’Arcy Thompson (Chorley, 1962).

Reliefs, formes, processus internes et externes... les choses pourraient être simple, en géomorphologie, ne seraient-ce la multiplicité des approches source de quiproquos et d'incompréhensions entre chercheurs, l'ambiguité s'attachant à de nombreux termes comme « relief » ou « surface d'érosion », à l'origine de bien des confusions, et, il faut le reconnaître, une invisibilité assez générale (Tooth, 2009) due à ce que la géomorphologie est souvent perçue non pas comme une fin en soi mais comme une boîte à outils ou une série de techniques au service par exemple de la géophysique ou de la géodynamique, comme la géographie le fut pour l'histoire au XIX ${ }^{\mathrm{e}}$ siècle avant la révolution vidalienne. Les choses se compliquent encore plus lorsque le questionnement morphologique porte sur les paysages, leurs perceptions (paysages visuels, sonores, olfactifs...), leurs représentations (gravures, tableaux, sculptures, photos, vidéos, installations...), sur les perceptions que l'on peut avoir tantôt d'un paysage donné, ou choisi, ou vécu, tantôt de sa et/ou de ses (re)présentation(s). Les perspectives sont ici sans limites, comme cet assemblage prolifique et protéiforme qu'évoque en 1866 Victor Hugo dans Les Travailleurs de la mer : « Une dynamique extraordinaire étale là ses problèmes, résolus. D'effrayants pendentifs menacent, mais ne tombent pas. On ne sait comment tiennent ces bâtisses vertigineuses. Partout des surplombs, des porte-à-faux, des lacunes, des suspensions insensées ; la loi de ce babélisme échappe ; l'inconnu, immense architecte, ne calcule rien, et réussit tout ; les rochers, bâtis pêle-mêle, composent un monument monstre ; nulle logique, un vaste équilibre ».

\footnotetext{
${ }^{6}$ « La question des questions se ramène à savoir où placer les limites entre la prédictibilité découlant des lois invariables et les multiples éventualités de la contingence historique » (Gould, 1991, 324).

${ }^{7}$ « Formes du relief et paysages dépendent des circonstances et sont les résultats contingents de lois déterministes opérant dans un environnement spécifique donné, de telle sorte que plusieurs possibilités existent ».
} 
La question, non du beau paysage en géographie ${ }^{8}$, mais de la beauté des formes du relief ou de celle des processus, porte la difficulté à son comble. Ruskin a dessiné les Aiguilles de Chamonix, Cézanne a peint la Sainte-Victoire, John Ford a filmé les buttes de Monument Valley et les méandres imprimés de la San Juan River. Les cascades de séracs gris-bleu des fleuves de glace, les sols figurés des domaines périglaciaires, le lac émeraude ${ }^{9}$, la mer turquoise, le sable blanc, le rougeoiement nocturne des laves, le flamboiement orangé des projections, le déferlement de la vague au pied de la falaise, le jaillissement de la cascade du haut de l'abrupt, l'écroulement mugissant des cataractes, les palettes de couleurs changeantes, les ruptures de pentes inattendues, le relief singulier énigmatique ou les lignes épurées à l'infini sont autant de dispositifs spectaculaires, pour lesquels le beau est invariablement convoqué : ainsi, dans Geomorphological landscapes of the world (Migoń, 2010) ${ }^{10}$, outre le Mont Fuji (Fig. 1) figurent les monolithes d'Uluru (Ayers Rock) et Kata Tjuta (Monts Olgas), les donjons quartzitiques (tepui) de la Gran Sabana du bouclier guyanais, les chutes de l'Iguaçu, ou du Zambèze à Victoria Falls (Mosi-oa-Tunya, « la fumée qui gronde »), les falaises du Dorset et du Devon oriental, les fjords de Norvège, les sols polygonaux du delta du Mackenzie, les pitons granitiques effilés de Sanqingshan et de Huangshan au sud-ouest de Shanghai, le karst à tourelles de Chine du Sud ou de Jamaïque, les atolls du Pacifique...

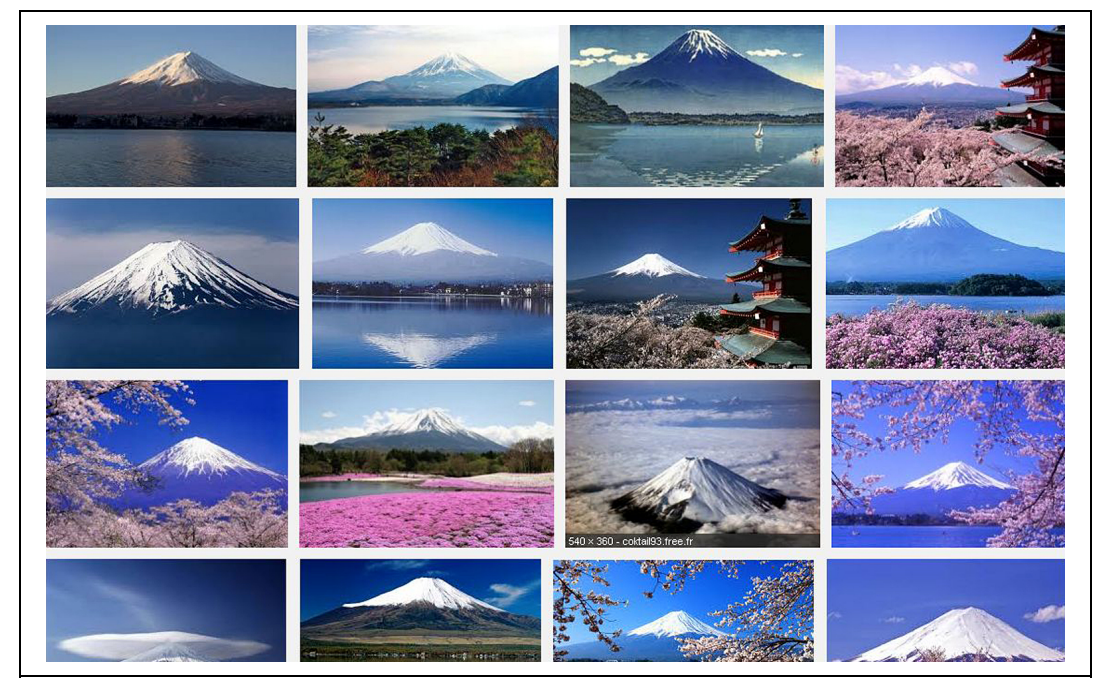

Figure 1 - Les seize premières images du Mont Fuji obtenues sur le web le 8 juin 2014 suite à la requête « Mont Fuji » (voir aussi page 26, in fine)

\footnotetext{
${ }^{8}$ Voir G. Neuray (1982), J.-R. Pitte (1983), A. Corbin (1988), J.-M. Besse (2000, 2009), Y. Luginbühl (2012). ${ }^{9} \mathrm{http} / / /$ en.wikipedia.org/wiki/Emerald_Lake_(Yukon) [consulté le 13 juin 2014]

${ }^{10}$ Un compte-rendu détaillé de l'ouvrage est disponible sous : http://geomorphologie.revues.org/8027 [consulté le 7 juin 2014]
} 


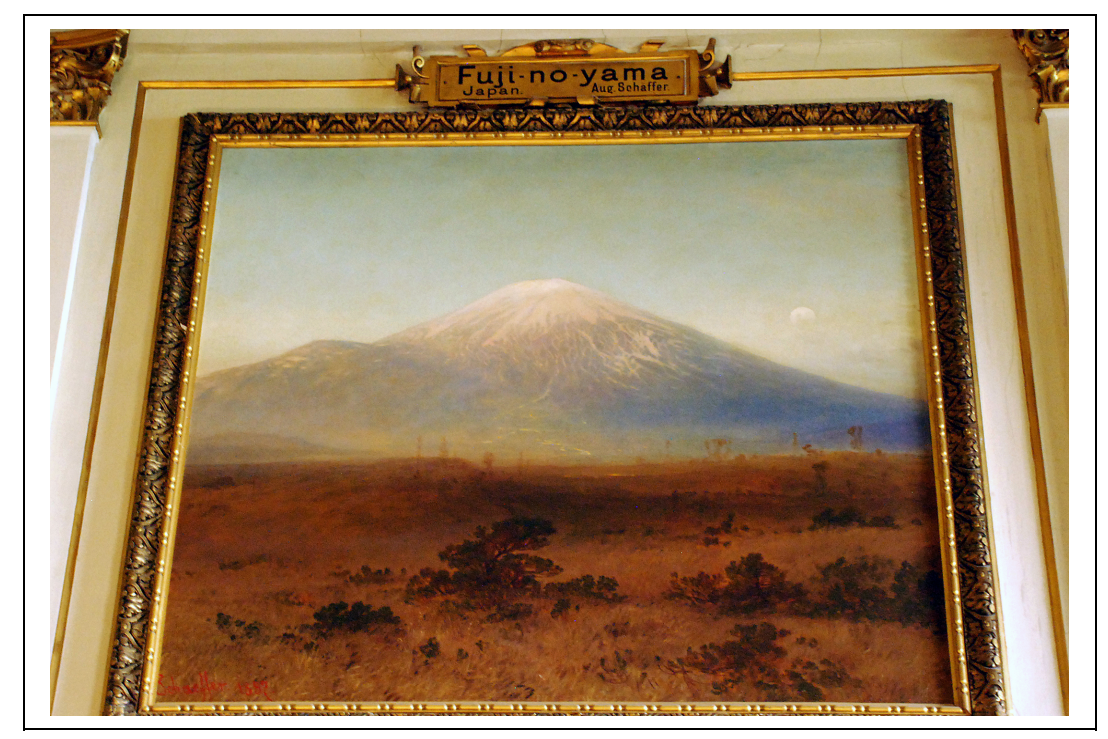

Figure 2 - Le « Fuji-no-yama » (Japon), peinture d'August Schaeffer (1887), Naturhistorisches Museum Wien. Photo prise le 30 avril 2014, (C) Christian Giusti

La fascination éprouvée pour le Mont Fuji ne date pas d'hier puisque l'emblématique volcan orne une salle du Muséum d'Histoire naturelle de Vienne (Fig. 2), ouvert au public en 1889, et à la décoration duquel ont participé de nombreux peintres paysagistes (Jovanović-Kruspel, 2003, 2012). La question est donc de comprendre, tant dans l'ordre des formes du relief que des variations du rapport d'intensité des processus internes et externes qui les engendrent, ce que pourrait être le « beau » en géomorphologie. Car, comme le remarquait Diderot dans ses Recherches philosophiques sur l'origine et la nature du beau (cité par Changeux, 2008) : «Comment se fait-il que presque tous les hommes soient d'accord qu'il y a un beau; qu'il y en ait tant d'entre eux qui le sentent vivement où il est, et que si peu sachent ce que c'est ? ». Jean Pelletier (1982) rappelle que « l'idéation esthétique ... change d'abord dans le temps avec les idéaux des sociétés de l'époque considérée, ... ensuite en une même époque par les différences, les variations induites par l'appartenance à des cultures, des classes sociales différentes ». D’autres éléments de réponse peuvent être trouvés chez des géomorphologues comme Luna B. Leopold (1969), un historien comme Alain Corbin (1988), ou des géographes comme Numa Broc $(1969,1991)$ qui, dans son étude sur la perception et la représentation des montagnes au siècle des Lumières, a montré comment les reliefs alpins et pyrénéens passent de l'affreux au sublime entre le XVII ${ }^{\mathrm{e}}$ et le $\mathrm{XIX}^{\mathrm{e}}$ siècle. - Une fois dissipées certaines ambiguïtés attachées au concept de relief, il devient possible d'esquisser une esthétique de la toposphère, afin d'interroger le statut des formes et processus du relief dans le regard que les sociétés occidentales contemporaines portent sur le paysage. 


\section{2. Énergie du relief - Formes du relief}

En français, le sens du mot « relief» s'est enrichi au cours du temps ${ }^{11}$. Dans Furetière (1690) ou dans la $2^{\mathrm{e}}$ édition du Dictionnaire de l'Académie (1718), le mot possède un sens juridique (droit de relief), mais est surtout employé en sculpture (haut-relief, bas-relief), en gravure (médailles), en joaillerie (montage des pierres), et en peinture (usage des ombres) : l'idée d'un objet en saillie se détachant sur un fond est déjà présente. Les « reliefs de table », pour désigner ce qui reste des viandes qu'on a servies ${ }^{12}$, est déjà d'un usage signalé comme vieux en 1740 (Acad., $3^{\mathrm{e}}$ éd.). La $4^{\mathrm{e}}$ édition du Dictionnaire (Acad., 1762) parle de l'élévation d'un plan ou «plan en relief » (plan relevé). Les éditions suivantes précisent le sens figuré (éclat), le sens propre (en mer, hauteur d'un navire sur l'eau ; à terre, hauteur d'un ouvrage au-dessus du terrain sur lequel il est bâti), les sens techniques (le clichage en imprimerie, le relief de la lettre). La synonymie avec « proéminent » apparaît en 1879 (Acad., $7^{\mathrm{e}}$ éd.) : « qui est plus en relief que ce qui l'environne ». Dans la $8^{\mathrm{e}}$ édition (Acad., 1932-1935), l'orographie est la partie de la géographie qui traite des montagnes, du relief terrestre ; surtout, le « relief » est « ce qui fait saillie sur une surface », les exemples étant : « le relief du sol», « le plan en relief d'une ville », « des lettres en relief » ou « le relief d'une médaille ».

« Relief du sol » a été employé par De Martonne dans toutes les éditions du Traité de géographie physique de 1909 à 1958 (Broc et Giusti, 2007). L'origine de cette expression vient moins d'un hypothétique partage des compétences (Giusti, 2012a) entre militaires (la topographie de surface, les formes du terrain), géologues (les topographies souterraines, le sous-sol, les mines) et géographes (le « relief du sol», les formes du relief), que de Vidal de La Blache qui, dans le Tableau de la géographie de la France, combine les sens pédologique et morphostructural du mot « sol », utilisant tantôt l'un ${ }^{13}$, tantôt l'autre ${ }^{14}$ (Giusti, 2012b). « Relief » possède aussi deux sens bien différents qui sont soigneusement distingués en anglais ou en allemand, mais pas en français où une expression comme « le relief de la France » peut renvoyer à la géomorphométrie (hypsométrie) ou à la géomorphologie.

\footnotetext{
${ }^{11}$ Toutes les références de ce paragraphe sont accessibles sur le site de l'INIST, Grand corpus des dictionnaires.

${ }^{12}$ Voir La Fontaine (1668), Le Loup et le Chien : «Moyennant quoi votre salaire / Sera force reliefs de toutes les façons : / Os de poulets, os de pigeons, / Sans parler de mainte caresse ».

${ }_{13}$ «Les plaines par lesquelles la Belgique confine à la France apparaissent [...] comme une contrée aussi uniforme par la nature des couches superficielles du sol que par le niveau général et le climat. Sur de grands espaces s'étendent des nappes limoneuses amortissant les inégalités du relief » (Vidal de La Blache, 1903).

${ }_{14}^{14}$ "C'est le même massif primaire qui, dans l'Ardenne comme dans les plaines qui s'y appuient au Nord-Ouest, constitue la charpente essentielle du sol » (Vidal de La Blache, 1903).
} 


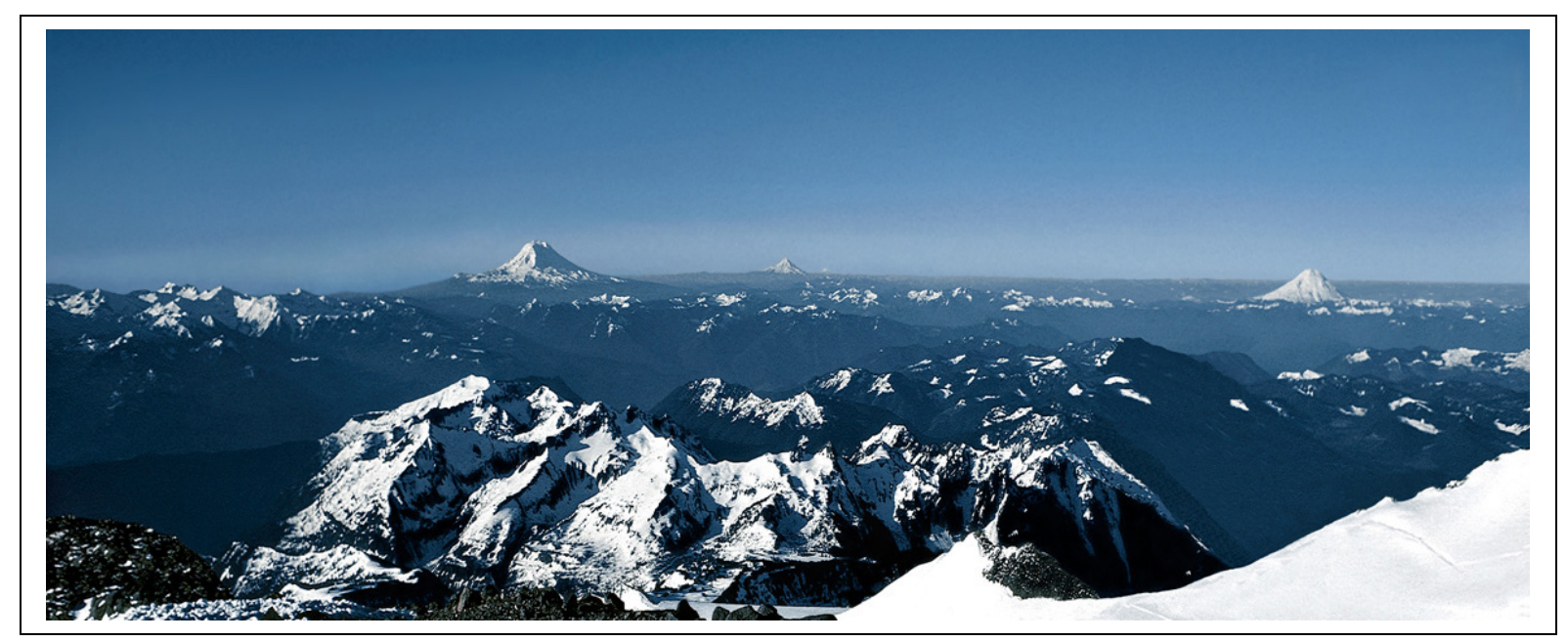

Figure 3 - Le Mont Adams, le Mont Hood et le Mont St Helens avant le 18 mai 1980, pris des pentes du Mont Rainier en direction du sud ${ }^{15}$

Relief désigne a priori tout volume faisant saillie en contre-haut d'un plan, par exemple les volcans de la chaîne des Cascades dominant le haut niveau de crêtes sub-égales ${ }^{16}$ - Gipfelflur ou accordance of summit levels - des topographies rugueuses subordonnées (Fig. 3). Tous ces reliefs pourront être caractérisés par la différence d'altitude entre points hauts et points bas (Reliefenergie, relief relatif, vigueur, énergie du relief, relief, locale relief, relative relief, relief index) : le sommet des volcans, l'altitude du niveau de crêtes, celle des fonds de vallée. L'oxymore « relief en creux » rappelle que certains ont voulu distinguer, par exemple à propos d'une topographie glaciaire d'abandon, knobs and lochans topography, le relief « négatif » d'une concavité qui abrite un lac opposé au relief « positif » des verrous à roches moutonnées. - Cependant, l'absence de relief caractérise près de $70 \%$ des terres émergées. Les formes volumiques ont une étendue moindre que les formes surfaciques, ces immenses horizons plans ou sub-horizontaux qui, des centres de bassin sédimentaire au cœur des boucliers, se distinguent par leur infinie monotonie, voire leur atonie, hormis quelques rares inselbergs : les « montinsules ». Toutes les dimensions, positives ou négatives, d'un volume de relief, quelle que soit sa surface, étant mesurables topographiquement par planimétrie et altimétrie (ou nivellement), l'attrait pour la morphométrie, jamais démenti depuis Sonklar et Albrecht Penck (Giusti, 2012b), a été plus que stimulé ces dernières années par l'essor de l'informatique (Hengl et Reuter, 2009) : la multiplication des études morphométriques, l'essor

\footnotetext{
${ }^{15} \mathrm{http}: / /$ www.earthwalkthebook.com/blog/getting-high [consulté le 16 juin 2014].

${ }^{16}$ Il est aujourd'hui établi que cette subégalité ne dérive pas de la dissection d'un aplanissement culminant de type « pénéplaine » (Davis), mais résulte plus probablement de l'espacement régulier des vallées et de l'équilibre dynamique établi régionalement entre facteurs internes et externes de la morphogenèse (Gilbert, Penck).
} 
de la télédétection active (Radar, Lidar) et la diffusion des MNT de précision métrique invitent à revisiter une vieille question : qu'est-ce qu'une forme du relief? (Evans, 2012).

Relief possède aussi un sens géomorphologique, les formes du relief (Oberflächenformen, landformen, landforms, relieffeatures), le « relief du sol » cher à de Martonne. Mais, alors que le relief, en tant que variable des topographies subaériennes ou sous-marines, est par définition continu (notion de continuum topographique, land surface form), les formes du relief (landform) sont des objets discontinus, aux limites tantôt floues, tantôt précises, mais avec des lignes, des surfaces, des volumes et, répondant à cette géométrie, des systèmes de pentes lisses et réguliers ou rugueux et accidentés, qui possèdent une signification et des propriétés. Celles-ci sont plus ou moins diversifiées selon les dimensions de l'objet, de la forme élémentaire à l'échelle du géotope (drumlin, doline, évent volcanique...) aux unités conceptuelles d'échelle globale comme la « surface africaine » de Lester C. King (Burke et Gunnell, 2008), en passant par tous les intermédiaires : géofaciès (versant, falaise, coteau...), géosystème (chaînon, vallée, volcan...), paysages-types (littoral, montagneux, continental...), régions naturelles ou provinces physiographiques (les Shetlands, les Pyrénées, l'Australie...).

Le paysage de la Chaîne des Cascades (Fig. 3) illustre deux propriétés fondamentales des formes du relief. Premièrement, le développement des formes du relief dans le temps n'est pas uniforme mais ponctué d'événements catastrophiques séparés par des phases de moindre activité, voire de quiescence. Cet aspect avait été clairement perçu par des chercheurs comme J Harlen Bretz à propos des Channeled Scabland (Baker, 2009) ou Walther Penck dans Die morphologische Analyse (Giusti, 2013), mais leurs propositions se sont heurtées à un mur de scepticisme presque total car, entre les deux guerres mondiales, la « science normale » (Kuhn, 1962) était une géomorphologie fondée sur le concept d'érosion «normale » (Klein, 1997). Deuxièmement, les formes du relief sont tout à la fois statiques et dynamiques : ici, tout dépend du repère spatio-temporel dans lequel le géomorphologue se place (Thornes et Brunsden, 1977). Ainsi, pour un observateur travaillant à l'échelle des chroniques (annuelle, mensuelle, journalière) et de l'enregistrement du temps instantané, le Mont Saint Helens passe de l'état « figé » avant son entrée en crise à l'état « explosif» au moment de la catastrophe du 18 mai 1980. Mais, hormis le cône, volatilisé dans l'explosion, et l'aire impactée par les retombées de cendres et les coulées boueuses de débris (lahars), les écosystèmes et les morphosystèmes de ce secteur de la chaîne des Cascades n'ont guère été modifiés. Pour un chercheur travaillant à des pas de temps de l'ordre du siècle à la dizaine de milliers d'années, 
ce sont les variations des paramètres climatiques qui ont le plus d'impact : isothermes, ligne d'équilibre des glaciers, limite des neiges persistantes, étages de végétation et systèmes morphogénétiques associés, anthropisation. Enfin, à l'échelle de la longue durée géologique, ou temps profond, pour des durées allant de la centaine de milliers à la centaine de millions d'années, les paramètres tectoniques (vitesse de subduction de la plaque Juan de Fuca sous la plaque nord-américaine) et eustatiques (variations positives ou négatives du niveau de l'océan Pacifique) seront les forçages majeurs. Le continuum topographique est donc très hétérogène au plan géomorphologique : il est un assemblage (2D), voire un feuilletage (3D) d'éléments ajustés aux conditions présentes de la morphogenèse (characteristic landforms), d'éléments en cours de ré-ajustement (transient landforms), ainsi que d'héritages anciens : paléoformes ou paléosols, régolithe, altérites, cuirasses... (Brunsden et Thornes, 1979. Bishop, 2007).

\section{Esthétique de la toposphère}

Dans Klima-Geomorphologie, qu'il dédie à ses maîtres Édouard Brückner et Albrecht Penck, Julius Büdel (1977) ${ }^{17}$ définit la Terrestrial relief sphere, qui comprend $90 \%$ de la surface libre de glace des terres émergées, «on which the higher plants and animals have evolved and which form the human habitat... »; et l'auteur de préciser que « still more important than the subaerial relief sphere are its four companion spheres ${ }^{18} »$, lesquelles « expand the relief sphere three-dimensionally ». Pour des raisons d'euphonie, R. Huggett a proposé de remplacer Relief sphere par Toposphere (Huggett et Cheesman, 2002), qui offre de plus l'avantage de pouvoir être directement transposé en français. La définition de la toposphère est simple : pour les terres émergées libres de glace, il s'agit de l'interface tri-dimensionnelle entre l'atmosphère et la lithosphère. Certes, ce concept ne résout pas le problème du flou ou de la précision des limites entre sous-ensembles, mais il intègre néanmoins le continu de la topographie (land surface form), le discontinu des formes du relief (landform), et l'épaisseur du palimpseste géomorphologique multi-couches (critical zone $)^{19}$, y compris dans ses composantes hydrologiques, pédologiques, biogéographiques, et anthropiques : «Humans have not only remade the features of the biological toposphere, they have also created new features for their own ends - buildings, bridges, roads, railways, canals, paths, fences, and so on » (Huggett et Cheesman, 2002). Les profondes transformations de la géographie des Émirats arabes unis depuis 1960 (Goudie et Viles, 2010, fig. 13) illustrent le phénomène de consommation des formes du relief pré-urbaines (Figure 4), comparables à d'autres mutations

\footnotetext{
${ }^{17}$ Citations en anglais, à partir de Climatic Geomorphology (Büdel, 1982).

${ }^{18}$ " The terrestrial hydrosphere and cryosphere, the biosphere, the pedosphere, the decomposition sphere ».

${ }^{19}$ Voir : http://www.czen.org/ [consulté le 19 juin 2014]. Mises au point in : H. Viles (2013), H. Wohl (2013).
} 
survenues après la Seconde Guerre mondiale en Amazonie, en Asie des moussons, ou en Chine continentale, humainement (mais non géologiquement) irréversibles.

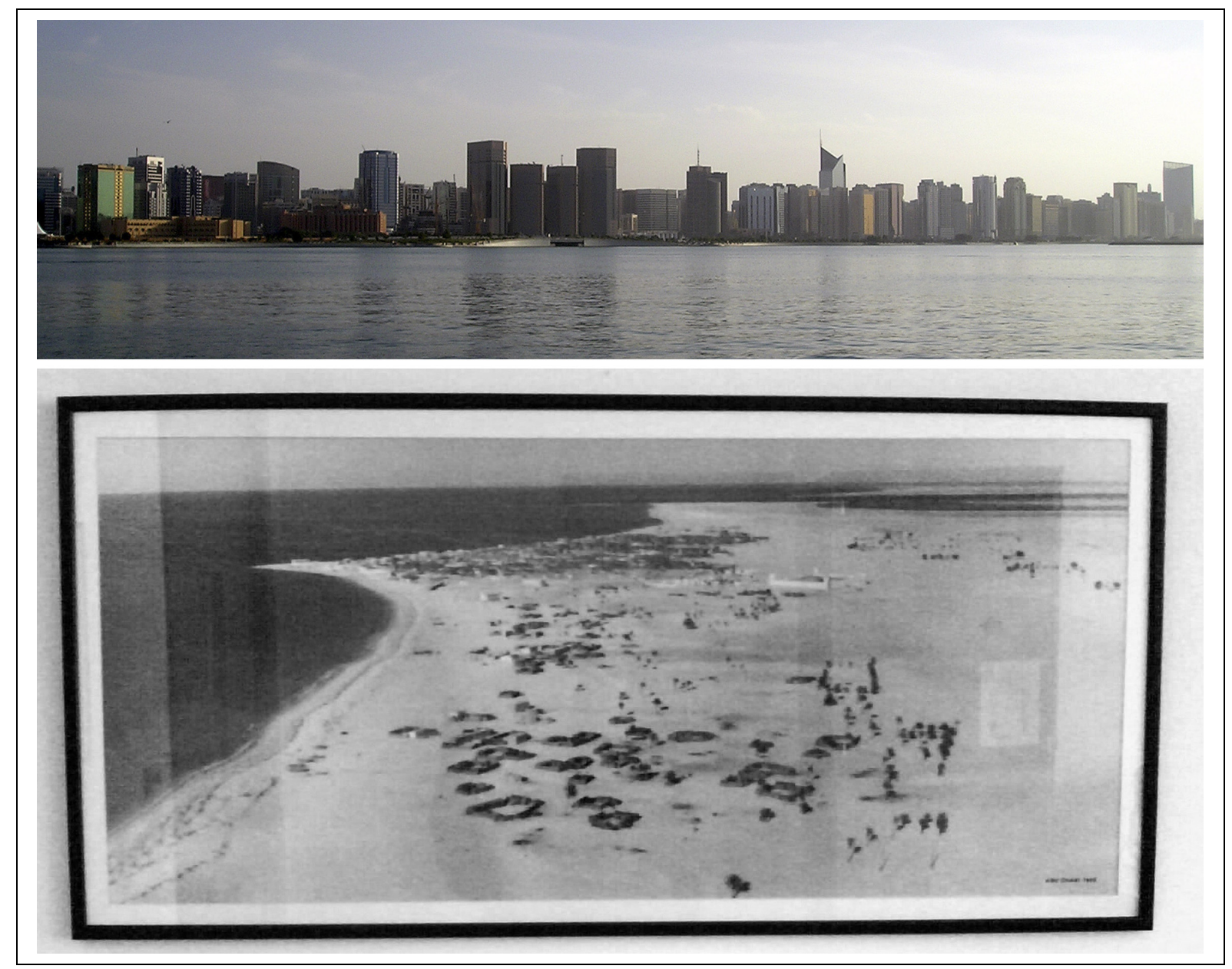

Figure 4 - La toposphère anthropisée : Abou Dhabi en 1955 et en $2007^{20}$

La complexité du continuum topographique avait était très nettement perçue par le géographe Pierre Birot (1955), pour qui tout géomorphologue était confronté à déchiffrer le palimpseste de l'épiderme terrestre en tenant solidement les deux bouts de la chaîne, des formes du relief aux processus de l'érosion, et réciproquement. En France, une géomorphologie centrée sur les formes et les cycles du relief (geographical cycle, denudation chronology) au détriment des processus a, sous l'empire de Davis, dominé la première moitié du $\mathrm{XX}^{\mathrm{e}}$ siècle. Au cours de la seconde moitié du $\mathrm{XX}^{\mathrm{e}}$ siècle, rares ont été les chercheurs qui, tels Claude Klein, ont continué de croire en l'autonomie des formes du relief et maintenu celles-ci au centre de leurs études. À l'image de personnalités comme Jean Tricart, Arthur Strahler, Julius Büdel, ou Richard

\footnotetext{
${ }^{20}$ Photos de l'auteur, prises en décembre 2007 depuis le port en direction de la Corniche (haut), et en 2010 dans un bureau du département de géographie à Paris-Sorbonne University in Abu Dhabi (bas).
} 
Chorley, l'immense majorité des géomorphologues s'est tourné vers une analyse approfondie des mécanismes morphogénétiques, adossée à l'essor de la quantification, de la théorie des systèmes, de l'informatique, de la télédétection, mais aussi de la tectonique globale, des analogues planétaires, de la paléoclimatologie, et du calibrage des courbes eustatiques (Schumm, 1991. Kennedy, 2006). Aussi la géographie du relief a-t-elle été un temps reléguée à l'arrière-plan de la physique et de la chimie du relief (Giusti, 2012a), avant de revenir sur l'avant-scène à partir des années 1990 du fait de l'intérêt renouvelé d'un nombre croissant de chercheurs pour ce qui, dans les paysages, présente un intérêt particulier au plan géomorphologique, processus actifs et spectaculaires ou formes monumentales et emblématiques (Panizza et Piacente, 2003. Reynard et al., 2009. Goudie et Viles, 2010). L'évolution scientifique de la géomorphologie n'est pas sans incidence sur l'idéation esthétique. Lorsque prévalait le paradigme davisien du geographical cycle, les surfaces géologiques de discordance angulaire et les surfaces d'aplanissement continentales (« pénéplaines ») étaient sur-valorisées au titre de témoin d'un ou de plusieurs cycles géomorphologiques. Aujourd'hui, les grandes chaînes de montagne de formation récente et les régions de tectonique vivante sont sur-valorisées au titre de laboratoires grandeur nature de l'interaction des mécanismes internes et des processus externes de la morphogenèse : ainsi, le volcan cesse d'être un « relief-postiche $»^{21}$ masquant les nobles vestiges du passé pour accéder au statut de système complexe doublement dynamique (éruptions, lahars...), dont l'actualité importe plus que l'histoire. D'objet emblématique de la tectonique des plaques et de la géomorphologie globale, le volcan acquiert, dans le cas du Mont Fuji et des nombreux autres volcans humanisés péri-pacifiques, la valeur de concept écouménal (Berque, 1999).

L'évaluation paysagère aux échelles locale ou régionale est devenue une thématique forte à partir du moment où les questions d'environnement ont commencé à envahir le champ social, et quand des implantations (pylônes et lignes électriques THT, tours de refroidissement, éoliennes) ou des activités (carrières, décharges, infrastructures de transport), se multipliant, ont eu tendance à être ressenties comme non-supportables (not in my backyard) par tout ou partie des populations concernées au quotidien, suscitant des mouvements dont l'idéologie prône successivement ou concurremment la protection, la préservation, la sauvegarde ou la conservation des milieux (Arnould et Glon, 2005). Comme l'a noté J.-L. Tissier (2003), « les acteurs sociaux ne portent plus sur le paysage un regard intéressé par la seule dimension

\footnotetext{
${ }^{21}$ Expression employée par De Martonne dans les éditions successives de Traité de géographie physique (Broc et Giusti, 2007).
} 
fonctionnelle. Ils considèrent qu'il est chargé de valeurs positives ou négatives, attractives ou répulsives, qu'il y a une relation entre leur état matériel et cet ordre de valeurs ». Dans les sciences du relief, c'est à la fin des années 1960 que des géomorphologues comme David Linton en Écosse (Lanarkshire) ou Luna Leopold sur la frontière de l'Idaho et de l'Oregon (Hell's Canyon, Snake River) se sont posés la question de la valeur esthétique des processus et des formes du relief (Panizza et Piacente, 2003). Linton (1968) s'appuie 2 catégories, le relief (Land Form) et l'utilisation du sol (Land Use) : pour la première, il fixe une échelle de valeurs comprenant six classes notées de 0 à 50 (plaine, plateau bas, plateau élevé, collines, collines élevées, montagnes); pour la seconde, ce sont sept classes notées de -25 à +30 (paysage industriel et urbanisé, forêt continue, campagne sans arbres, lande en plaine ou sur collines, lande et forêt diversifiées, campagnes très diversifiée, paysage sauvage). Testée dans la région des Lomond Hills, au nord-ouest de Glasgow, cette méthode a tendance à privilégier les formes du relief (pas de notes négatives) et les fortes altitudes (relief relatif), au détriment du couvert végétal et d'autres formes d'utilisation du sol (Gilg, 1976. Flatrès-Mury, 1982). Leopold (1969) étudie 3 catégories intégrant 46 facteurs : 14 facteurs physiques, de la largeur de la rivière, sa profondeur, sa vitesse, ... à l'érosion des berges et à la largeur de la vallée ; 14 facteurs biologiques et hydrologiques comme la couleur, la turbidité, la faune, la flore, la pollution des eaux, ... jusqu'à la nature de la couverture végétale et à l'état général de la vallée ; 18 facteurs socio-économiques comme la présence ou l'absence de déchets, de barrages, de voies d'accès, de vues panoramiques, d'incongruités (misfits), ... jusqu'à l'ouverture du paysage, à l'utilisation du sol, au niveau d'équipement et d'urbanisation, et aux particularités historiques. La méthode permet d'évaluer de façon quantitative la valeur esthétique d'un paysage (est-il banal ? au-dessus de la moyenne ? exceptionnel ?), en calculant ce que ce paysage présente d'unique dans sa globalité (total uniqueness ratio, TUR), et ce que valent des combinaisons de facteurs aux échelles subordonnées de la vallée (valley character) ou de la rivière (river character). Appliquée à 12 sites du bassin de la Snake River aux limites de l'Idaho et de l'Oregon, la méthode montre que Hell's Canyon sur la Snake et Carey Falls sur la Salmon River sont des lieux remarquables à toutes les échelles : du géosystème (le paysage) au géofaciès (la vallée, la rivière) et au géotope (le lieu), si l'on met en parallèle la grille de lecture de Leopold (1969) avec la terminologie de G. Bertrand (1968). Appliquée aux États-Unis, elle place Hell's Canyon au deuxième rang juste après le Grand Canyon du Colorado (Arizona), mais loin devant la Merced River du Parc national de Yosemite dans la Sierra Nevada (Californie). 
Ces essais de quantification de la valeur esthétique des composantes géomorphologiques du paysage sont contemporains de l'essor du courant aménagiste qui, dans la deuxième moitié du $20^{\mathrm{e}}$ siècle et au nom d'une logique d'exploitation effrénée des milieux, symbole de modernité, vise à toujours repousser les limites de l'œkoumène, tant en Occident que dans la sphère d'obédience marxiste. En 1959, la décision du gouvernement égyptien de barrer le Nil à Assouan entraîna de la part de l'UNESCO le lancement d'un programme de sauvetage des temples d'Abou Simbel auquel participèrent plus de 50 États souverains. Les inondations de Venise et de Florence (Italie) en novembre 1966, les opérations de sauvegarde des sites de Carthage (Tunisie) et de Borobudur (Indonésie) en 1970, renforcèrent le sentiment d'une nécessaire coopération internationale pour faire face à des situations où un patrimoine de valeur exceptionnelle et universelle se retrouverait en situation de grand péril. Trois traités internationaux étaient en cours de gestation à la fin des années 1960, l'un sur les monuments historiques à l'initiative de l'UNESCO ${ }^{22}$, l'autre sur le patrimoine naturel à l'initiative de l'Union internationale pour la conservation de la nature (UICN) ${ }^{23}$, un groupe de travail créé en $1968^{24}$ étudiant simultanément la possibilité de combiner les deux projets en un seul texte. C'est ainsi que vit le jour en novembre 1972 la Convention du patrimoine mondial, World Heritage Convention (Depraz, 2008. Francioni et Lenzerini, 2008. Forrest, 2010).

Convention du patrimoine mondial - Définition du patrimoine culturel et naturel

World Heritage Convention - Definition of Cultural and Natural Heritage

Article 1

Aux fins de la présente Convention sont considérés comme

" patrimoine culturel »:

les monuments : œuvres architecturales, de sculpture ou de peinture monumentales, éléments ou structures de caractère archéologique, inscriptions, grottes et groupes d'éléments, qui ont une valeur universelle exceptionnelle du point de vue de l'histoire, de l'art ou de la science,

les ensembles: groupes de constructions isolées ou réunies, qui, en raison de leur architecture, de leur unité, ou de leur intégration dans le paysage, ont une valeur universelle exceptionnelle du point de vue de l'histoire, de l'art ou de la science,

les sites: œuvres de l'homme ou œuvres conjuguées de l'homme et de la nature, ainsi que les zones y compris les sites archéologiques qui ont une valeur universelle exceptionnelle du point de vue historique, esthétique, ethnologique ou anthropologique.

Article 2
«ux fins de la présente Convention sont considérés comme
« patrimoine naturel »:
les monuments naturels constitués par des formations physiques et
biologiques ou par des groupes de telles formations qui ont une
valeur universelle exceptionnelle du point de vue esthétique ou
scientifique,
les formations géologiques et physiographiques et les zones
strictement délimitées constituant l'habitat d'espèces animale et
végétale menacées, qui ont une valeur universelle exceptionnelle
du point de vue de la science ou de la conservation,
les sites naturels ou les zones naturelles strictement délimitées, qui
ont une valeur universelle exceptionnelle du point de vue de la
science, de la conservation ou de la beauté naturelle.

Tableau 1 - Les articles 1 et 2 de la Convention du patrimoine mondial (1972)

La Convention est divisée en 38 articles $^{25}$, qui concernent surtout les moyens de protection du patrimoine mondial. La notion cardinale est celle d' " outstanding universal value » (OUV),

\footnotetext{
${ }^{22}$ International Protection of Monuments, Groups of Buildings and Sites of Universal Value.

${ }^{23}$ Convention for the Conservation of the World's Heritage.

${ }^{24}$ The United Nations conference on the Human Environment.

${ }^{25} \mathrm{http}: / /$ whc.unesco.org/fr/conventiontexte [consulté le 10 juillet 2014].
} 
cette « valeur universelle exceptionnelle » (VUE) omniprésente (Tabl. 1), associée à l'adjectif « esthétique » et à l'expression « beauté naturelle » dans la définition tant du patrimoine culturel (cultural heritage, art. 1) que naturel (natural heritage, art. 2). Parce que son contenu peut changer suivant les époques et selon les aires culturelles, la VUE n'est pas définie dans la Convention mais dans les « Orientations devant guider la mise en œuvre de la Convention» (Operational Guidelines), régulièrement mises à jour depuis leur première édition en $1977^{26}$, afin de corriger certains déséquilibres initiaux (sur-représentation des monuments au détriment des paysages, de la chrétienté par rapport aux autres religions...). La VUE est donc le type même de la notion en devenir (evolving concept), dont les infléchissements successifs ont permis l'émergence des concepts de « patrimoine mixte culturel et naturel» (§46) et de « paysage culturel» (§ 47), qui prend en compte « les œuvres conjuguées de l'homme et de la nature » (Tabl. 1). Dans la version 2013 des « Orientations », la VUE fait l'objet des paragraphes $49-53$ et 154 : ainsi, le $\S 49$ stipule que « la valeur universelle exceptionnelle signifie une importance culturelle et/ou naturelle tellement exceptionnelle qu'elle transcende les frontières nationales et qu'elle présente le même caractère inestimable pour les générations actuelles et futures de l'ensemble de l'humanité ». Les critères d'évaluation de la VUE sont définis aux $\S \S 77-78^{27}$, (i) à (vi) pour le patrimoine culturel (dont les biens doivent aussi satisfaire aux conditions d'authenticité définies aux $\S \S 79-86$ ), (vii) à (x) pour le patrimoine naturel. Enfin, tous les biens proposés doivent également répondre à des conditions d'intégrité ( $\S 87-90)$, précisées pour chaque critère ( $\S 91-95)$ : en particulier, dans le cas des biens proposés selon le critère (vii), « représenter des phénomènes naturels ou des aires d'une beauté naturelle et d'une importance esthétique exceptionnelles » (§ 78), ils « doivent être d'une valeur universelle exceptionnelle et inclure des zones essentielles au maintien de la beauté du site. C'est ainsi qu'un site auquel une chute d'eau conférerait des valeurs esthétiques, répondrait aux conditions d'intégrité s'il incluait également le bassin qui l'alimente ainsi que des aires en aval intégralement liées au maintien des qualités esthétiques du site » (§92). On citera ici des biens comme les chutes d'Iguazú sur la frontière BrésilArgentine, ou Mosi-oa-Tunya (Victoria Falls) sur la frontière Zambie-Zimbabwe.

\footnotetext{
${ }^{26} \mathrm{http} / / /$ whc.unesco.org/fr/orientations/ [consulté le 11 juillet 2014].

${ }^{27}$ Voir aussi : http://whc.unesco.org/fr/criteres/ [consulté le 10 juillet 2014].
} 


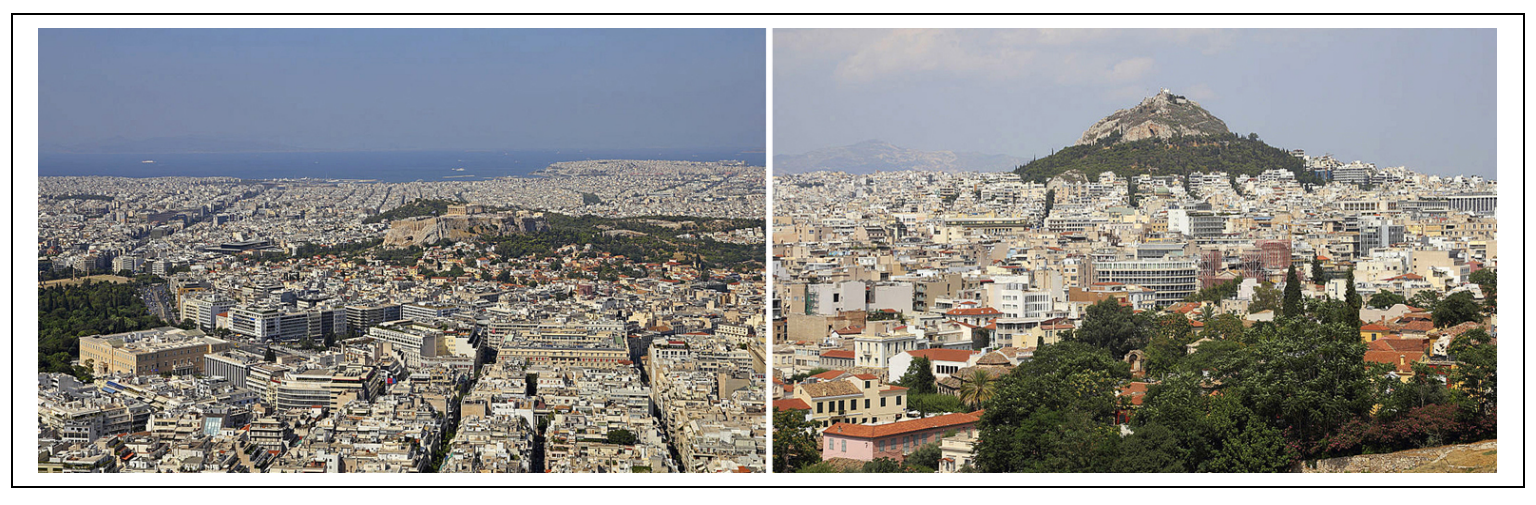

Figure 5 - Étalement de la nappe bâtie et encerclement d'îlots de reliefs à Athènes (Grèce). Athènes et Le Pirée vus du Lycabette (gauche) ; le Lycabette vu de l'Acropole (droite) ${ }^{28}$.

\section{Traits géomorphologiques des paysages de l'Anthropocène}

Le concept d'Anthropocène a été proposé pour désigner une période au cours de laquelle l'influence des êtres humains sur le géosystème est devenue prédominante. Certains font commencer cette période dès la préhistoire, soit à la fin des grandes glaciations pléistocènes, soit pendant l'Holocène, avec l'essor des agricultures. D'autres estiment que la révolution industrielle et l'invention de la machine à vapeur signent le début de l'Anthropocène stricto sensu, une phase marquée pour l'humanité par l'accélération de la croissance démographique, l'urbanisation, la démultiplication des forces motrices et l'essor des réseaux d'information, mais aussi par l'accumulation universelle des déchets de toute nature, d'où l'étiquette « Poubellien supérieur » reprise par le géologue du Muséum P. De Wever (2012) pour stigmatiser cette particularité (Eugène-René Poubelle a été préfet de la Seine de 1883 à 1896). Alors qu'en 1890, seule la Grande-Bretagne dépassait le seuil de 50\% de population urbaine, à l'horizon 2025 (donc demain), le taux d'urbanisation sera de $61 \%$ en moyenne mondiale, de 54 à 55\% en Afrique et en Asie, de 75\% en Océanie, de 83 à $85 \%$ en Amérique et en Europe. Les villes, les agglomérations, les régions urbaines, les méga-régions urbaines, l'archipel métropolitain ouest-européen, la Megalopolis nord-américaine, la ceinture Beijing-SeoulTokyo (BESETO), voire la « ville-monde » (ecoumenopolis) prédite par l'architecte et urbaniste grec Constantin Doxiadis pour la fin du XXI ${ }^{\mathrm{e}}$ siècle (Pacione, 2009), sont tous des organismes consommateurs de relief par asphaltage, barrage, chenalisation, creusement, forage, imperméabilisation, nivellement, remodelage, ou terrassement, mais principalement par étalement spatial (urban sprawl) (Figure 5). De nos jours, l'Acropole d'Athènes et les collines du Lycabette ou de la Pnyx ne sont plus que des îlots de relief autochtones émergeant de l'empilement des nappes de constructions superposées, sous lesquelles s'interpose pour le

\footnotetext{
${ }^{28}$ Photos d'Alexander Savin (2013) dans « History of Athens », source Wikimedia Commons.
} 
plus grand bonheur des archéologues une semelle plus ou moins épaisse de déchets, déblais, et remblais, masquant le tréfonds rocheux taraudé. À Rome (Pica et al., 2014) comme à Paris (Kennel, 2013), un épais film d'immeubles, ici adhère au relief des Sept collines, feutrées, là masque le col de la Chapelle, que nul ne distingue plus entre la butte Montmartre et les hauteurs de Belleville. Du haut de la tour Montparnasse, les dominos du Front-de-Seine, l'iconique tour Eiffel et la skyline de La Défense diluent la perception des bord de Seine et du Mont Valérien : l'énergie du relief des tours égale ou surpasse celle des buttes et des coteaux (Tableau 2). En valeur absolue, c'est un relief artificiel, la colline d'Élancourt, qui porte le point culminant de la région Île-de-France, où l'épaisseur de la toposphère anthropisée est de l'ordre de $1 \mathrm{~km}$, des 800 millions de $\mathrm{m}^{3}$ de gaz naturel stockés à $-740 \mathrm{~m} / \mathrm{sol}$ au réservoir profond de Beynes (Yvelines), à $+324 \mathrm{~m} / \mathrm{sol}$ à la pointe des antennes installées au sommet de la tour Eiffel (la troposphère inférieure étant elle-même substantiellement affectée par les activités humaines).

\begin{tabular}{|l|c|c|c|c|c|c|c|}
\hline Site & Localisation & Objet & $\begin{array}{c}\text { Altitude } \\
\text { de la base }\end{array}$ & $\begin{array}{c}\text { Nature de } \\
\text { la base }\end{array}$ & $\begin{array}{c}\text { Altitude } \\
\text { du } \\
\text { sommet }\end{array}$ & $\begin{array}{c}\text { Nature } \\
\text { du } \\
\text { sommet }\end{array}$ & $\begin{array}{c}\text { Relief } \\
\text { relatif } \\
\text { (arrondi) }\end{array}$ \\
\hline Colline d'Élancourt & Élancourt (78), France & Butte & $170 \mathrm{~m}$ & Espace vert & $231 \mathrm{~m}$ & Déblais & $60 \mathrm{~m}$ \\
\hline Front-de-Seine & Paris (75), France & Immeubles & Vers $30 \mathrm{~m}$ & Bâti & $128 \mathrm{~m}$ & Bâti & $100 \mathrm{~m}$ \\
\hline Mont Valérien & Suresnes (92), France & Butte, fort & Vers $30 \mathrm{~m}$ & Bâti & $161 \mathrm{~m}$ & Bâti & $130 \mathrm{~m}$ \\
\hline Tour Montparnasse & Paris (75), France & Immeuble & $53 \mathrm{~m}$ & Bâti & $262 \mathrm{~m}$ & Bâti & $210 \mathrm{~m}$ \\
\hline Tour First & Courbevoie (92), France & Immeuble & Vers $30 \mathrm{~m}$ & Bâti & $261 \mathrm{~m}$ & Bâti & $230 \mathrm{~m}$ \\
\hline Tour Eiffel & Paris (75), France & Édifice & $33 \mathrm{~m}$ & Bâti & $357 \mathrm{~m}$ & Antennes & $324 \mathrm{~m}$ \\
\hline Buttes coquillières & $\begin{array}{c}\text { St-Michel-en-L’Herm } \\
\text { (85), France }\end{array}$ & Édifice & 2 à $3 \mathrm{~m}$ & Marais & 6 à $11 \mathrm{~m}$ & Végétation & $<10 \mathrm{~m}$ \\
\hline $\begin{array}{l}\text { Parc éolien du } \\
\text { Chemin d'Ablis }\end{array}$ & $\begin{array}{c}\text { Fresnay-l'Évêque } \\
\text { (28), France }\end{array}$ & $\begin{array}{c}\text { Plateau, } \\
\text { équipement }\end{array}$ & Vers $130 \mathrm{~m}$ & Champ & 244 à $276 \mathrm{~m}$ & Pale & 110 à $150 \mathrm{~m}$ \\
\hline Grande Pyramide & Gizeh, Égypte & Édifice & $230 \mathrm{~m}$ & Plateau & $370 \mathrm{~m}$ & Bâti & $140 \mathrm{~m}$ \\
\hline Burj Khalifa & $\begin{array}{c}\text { Dubaï, } \\
\text { Émirats Arabes Unis }\end{array}$ & Immeuble & $<10 \mathrm{~m}$ & Bâti & $829,8 \mathrm{~m}$ & Antenne & $830 \mathrm{~m}$ \\
\hline
\end{tabular}

Tableau 2 - Relief relatif de différents objets en surface de la toposphère francilienne et éléments de comparaison (plateau, butte, édifices, équipements, immeubles)

Dans de nombreux pays du monde, le phénomène d'étalement entraîne une urbanisation sans limite spatiale, pilotée par la seule logique du profit et de la rentabilité au détriment de la vie sociale, du domaine rural et de l'environnement naturel (Pacione, 2009). L'urban sprawl n'est pas sans impact sur l'esthétique des formes du relief, plus ou moins masquées ou impactées par les formes urbaines et les infrastructures associées. Les reliefs urbains (intra-urbains, périurbains, et des edge cities, les pôles suburbains) présentent des formes couvertes, bâties à plus de $80 \%$ de leur superficie. Les reliefs suburbains (ceintures limitrophes, fringe belts, et edgeless cities, aux formes urbaines peu denses) se caractérisent par un taux de couverture variable, pouvant aller de 20 à $80 \%$, mais l'essentiel est ici la production d'étendues 
passablement hétéroclites où le continuum topographique se résout en discontinuités (géo)morphologiques, juxtaposant bretelles d'autoroutes, parcs de stationnement, lotissements résidentiels, centres commerciaux, immeubles de bureaux, stades de football, hippodromes, terrains de golf, et fragments d'espaces plus ou moins naturels. Enfin, les reliefs exo-urbains sont ceux où, dans le paysage, la dénaturation des composantes géomorphologiques (formes et processus) vient moins des surfaces bâties que de la prolifération des objets incongrus (antennes, barrages, carrières, châteaux d'eau, monuments militaires ou religieux, parcs éoliens, pylônes, radars, tours de télécommunication), des zébrures de lignes de transport de force électrique (HT, THT), et des balafres des infrastructures de transport (corridors autoroutiers et ferroviaires, réseau routier et pistes forestières, télécabines et téléphériques).

Mais les sociétés humaines ne se limitent pas à bâtir le relief ${ }^{29}$ ou à modifier la dynamique des fleuves (barrages) et des glaciers (réchauffement climatique) : Homo sapiens est aussi un redoutable agent d'érosion en bandes organisées, aux moyens mécaniques exacerbés depuis le début de la révolution industrielle, et grandement accélérés depuis 1950 (Steffen et al., 2007). La capacité actuelle de certains engins de chantier (excavateurs à godets de plus de 100 tonnes et camions-bennes de 400 tonnes) permet de les comparer à des armes de destruction massive au plan géomorphologique, comme dans le cas de l'exploitation des sables bitumineux des champs de Peace River, Athabasca ${ }^{30}$, et Cold Lake (Alberta, Canada), ou du nivellement de 700 sommets de montagnes dans la région de Lanzhou (province du Gansu, Chine). Les engins de dragage commettent des dégâts de même nature dans les lits fluviaux (ballastières) et sur la plateforme continentale, par exemple dans le cas des bancs de sables pré-littoraux, dont l'exploitation impacte l'équilibre des plages. Selon les mesures réalisées à la fin du XX siècle (Hooke, 1994, 2000), l'érosion anthropique liée aux seules pratiques agricoles pèse 70 giga-tonnes $(\mathrm{Gt})$, presque autant que la masse déplacée par les agents d'érosion « naturels » (cours d'eau, glaciers, processus éoliens, littoraux et de versant). Tous facteurs confondus (agriculture, immobilier, routes et autoroutes, mines et carrières), les actions anthropiques pèsent $100 \mathrm{Gt}$, contre $75 \mathrm{Gt}$ pour les agents non-anthropiques, et il est désormais acquis que le phénomène a commencé dès l'Holocène, voire plus tôt (Wilkinson, 2005. Neboit, 2010). Aussi certains services accordent-ils une attention de plus en plus soutenue aux formations géologiques artificielles d'origine anthropiques, comme en Grande-Bretagne (McMillan et

\footnotetext{
${ }^{29}$ Toute installation d'éolienne s'accompagne de la mise en place de socles en béton armé de 800 à 2000 tonnes, ancrés jusqu'à 20 mètres de profondeur.

${ }^{30} \mathrm{La}$ zone exploitable a été élargie à 51 municipalités en $2009\left(\right.$ soit $\left.4700 \mathrm{~km}^{2}\right)$, ce qui amène localement la frontière du champ à $19 \mathrm{~km}$ du parc national Wood Buffalo, un bien du patrimoine mondial.
} 
Powell, 1999) où cinq groupes sont distingués sur la nouvelle édition Superficial Deposits des cartes géologiques détaillées $^{31}$ : les formations construites (digues, terrils...), les déblais (tranchées routières, carrières...), les remblais, les formations terrassées paysagères (remodelage du paysage), les formations hétérogènes (lorsqu'il n'est pas possible de distinguer clairement l'un ou l'autre des types précédents). Les bases d'une stratigraphie de l'Anthropocène sont posées (Waters et al., 2014), un champ de recherche auxquels les géographes sont habilités à contribuer, notamment en ce qui concerne les dépôts et les formes de surface ou de subsurface d'origine anthropique (géoarchéologie, archéogéographie).

Les composantes géomorphologique des paysages de l'Anthropocène sont de moins en moins « naturelles », la question étant d'apprécier le degré de transformation anthropique par rapport à un état de référence, que celui-ci soit le milieu du XVIII ${ }^{e}$ siècle ou le milieu de l'Holocène : ici, l'étude de l'iconographie couplée à celle des paléoenvironnements s'avère fondamentale. Si la croissance de l'humanité en tant qu'espèce biologique est sans impact notable sur le fonctionnement des grands forçages internes (tectonique et magmatisme), il en va autrement à la surface de la toposphère (constructions et érosions anthropiques) et dans la basse atmosphère (changement climatique), où l'état des reliefs (lignes, surfaces, volumes) et le fonctionnement des grands forçages externes (ambiances climatiques, cours d'eau, glaciers, météorisation) sont impactés à des degrés divers dans l'espace et dans le temps.

\section{5. Épilogue}

Les sites d'intérêt géomorphologique ou géomorphosites (Panizza, 2001) diffèrent des autres sites d'intérêt géologique (géosites stratigraphique, paléontologique, minéralogique...) par trois propriétés spécifiques : la beauté des formes (critère esthétique), la dynamique des processus géomorphologiques (internes ou externes), et leur nature multi-scalaire liée, d'une part à l'emboîtement des échelles spatiales (géotope, géofaciès, géosystème), d'autre part à leur structure de palimpseste multi-couches (formes et modelés, formations superficielles et héritages). À l'échelle des objets (géotope), la dimension esthétique résulte souvent d'une allure inhabituelle (blocs et boules des pays granitiques, rochers singuliers mimant une forme humaine ou animale...), d'une couleur particulière (granite rose, marbre blanc, terres noires, pélites rouges...), du spectacle de l'érosion sélective (pinacles des reliefs karstiques, cheminées de fée, cascade sciant un verrou, sources vauclusiennes...), d'une localisation

\footnotetext{
${ }^{31}$ Le British Geological Survey établit et publie deux cartes distinctes par feuille : les anciennes séries Solid ou Drift sont progressivement remplacées par les nouvelles séries Bedrock ou Superficial Deposits. En France, le projet de cartographie du régolithe piloté par le BRGM représente une initiative de même nature.
} 
spatiale inattendue et contrastante par rapport à l'environnement géomorphologique (bloc erratique, cratère d'impact, inselberg...). Ces curiosités sont généralement connues de longue date et, dans certains cas, protégées en tant que « monument naturel » (Reynard et al., 2009). À l'échelle du paysage (géofaciès ou géosystème), la dimension esthétique est aussi très souvent une valeur essentielle du géomorphosite (paysages dits « de carte postale », type « La Pointe du Raz » ou « Le Mont-Blanc »), soit depuis un point de vue isolé (table d'orientation, sommet remarquable, belvédère aménagé...), soit à partir des points de vue multiples et complémentaires d'un réseau de sites (disconnected geomorphosite) (Reynard, 2009), soit depuis un géospot dans le cas où un géotope est couplé à une vue majeure de type panorama (Giusti et al., 2013). À ces échelles, du fait des transformations plus ou moins importantes survenues dans le plan de la toposphère, notamment dans les parties du monde exposées à des formes d'occupation humaine anciennes, persistantes, et plurielles, il est difficile voire impossible de trouver des paysages « naturels » : le plus souvent, ceux-ci sont le support d'activités variées, liées à l'économie, aux transports et à l'urbanisation. Dans une certaine mesure, les catégories Ia et Ib de l'UICN (Réserve naturelle intégrale, Zone de nature sauvage) et, en France, le cœur des parcs nationaux, sont des aires géographiques protégées où les composantes géomorphologiques du paysage conservent cet aspect « naturel».

En juillet 2014, la liste du patrimoine mondial comptait 1007 biens répartis dans 161 États, 779 au titre du patrimoine culturel (77\%), y compris les « paysages culturels » (pour « les œuvres conjuguées de l'homme et de la nature »), contre 197 au titre du patrimoine naturel (19\%), et 31 biens « mixtes » (4\%) comme le Tassili n'Ajjer (Algérie), le parc d'Uluru - Kata Tjuta (Australie), les Météores et le Mont Athos (Grèce). Sans surprise, les pays possédant le plus grand nombre de biens culturels sont - notamment - l'Allemagne, la Belgique, le Brésil, la Chine, l'Espagne, la France, la Grèce, l'Inde, l'Iran, l'Italie, le Japon, le Mexique, le Portugal, la Pologne, la Russie, la Suède, la Turquie, ou le Royaume-Uni. À l'opposé, les pays possédant le plus grand nombre de biens naturels sont souvent des États-continents comme l'Australie, le Brésil, le Canada, la Chine, les États-Unis, l'Inde, ou la Russie. Dans ce cas, nombreux sont les sites où les composantes géomorphologiques jouent un rôle important, comme dans (liste non exhaustive) la mer des Wadden (Allemagne, Danemark, Pays-Bas), le parc national Los Glaciares (Argentine), les Montagnes Bleues, le massif gréseux de Bungle Bungle et la Côte de Ningaloo (Australie), le delta de l'Okavango (Botswana), les parcs des Montagnes Rocheuses canadiennes, le karst de Chine du Sud, les parcs nationaux de Grand Canyon et Yosemite (États-Unis), le lac Baïkal et les volcans du Kamchatka (Russie), le 
Golfe de Porto et l'île de La Réunion (France), les Dolomites et le Mont Etna (Italie), les lacs du Kenya dans la vallée du Grand Rift, le Tsingy de Bemaraha (Madagascar), l'erg du Namib, les fjords de l'ouest de la Norvège, la Chaussée des Géants ou le littoral du Dorset et du Devon oriental (Royaume-Uni), les Alpes suisses Jungfrau-Aletsch, les lacs d'Ounianga (Tchad), la baie d'Ha-Long (Viet Nam) et les chutes Victoria (Zambie, Zimbabwe).

Or, le concept de géomorphosite (Panizza, 2001), qui réunit les deux dimensions de la science des reliefs, les formes (le cône volcanique, la vallée en auge...) et les processus, internes ou externes (l'éruption magmatique, le creusement du glacier de vallée...), intègre aussi les deux dimensions du beau en géomorphologie : la compréhension des phénomènes à travers la valeur scientifique centrale (la tectonique des plaques, l'érosion glaciaire...), et la dimension esthétique en tant que valeur additionnelle (pureté des lignes du cône, spectacle de la fontaine de lave ou du panache de cendres, beauté des glaciers crevassés et des cascades de séracs, géométrie en $U$ de la vallée en auge...). Toutefois, la valeur esthétique d'un géomorphosite ne devrait jamais masquer sa valeur scientifique (Reynard et al., 2009), ni le beau en géomorphologie être réduit à la seule esthétique des formes ou des processus : au-delà de la société du spectacle, l'élégance de la démonstration et la clarté des explications ont également droit à un haut statut. La beauté d'un site peut avoir pour intérêt de stimuler le tourisme et de procurer des ressources en générant des activités mais, comme le montre l'exemple du réseau des géoparcs en Europe et dans le monde ${ }^{32}$, elle est aussi une formidable porte d'entrée pour éduquer les citadins aux difficiles questions de géographie environnementale.

\section{Remerciements}

Cet essai doit beaucoup à Hervé Régnauld, qui m'a orienté vers l'esthétique des reliefs, à Gérard Hugonie, qui m'a fait découvrir les travaux d'Alain Corbin, et à Guy Chemla qui, en me confiant l'enseignement de géographie physique appliquée à l'aménagement du territoire, a guidé une part de mes réflexions vers les thèmes de la géomorphologie urbaine.

\footnotetext{
${ }^{32}$ http://www.europeangeoparks.org/ et http://www.globalgeopark.org/ [consultés le 18 juillet 2014].
} 


\section{Bibliographie}

Anderson, Robert, Anderson, Suzanne, (2010), Geomorphology. The Mechanics and Chemistry of Landscapes, Cambridge, CUP.

Arnould, Paul, Glon, Éric, (2005), La nature a-t-elle encore une place dans les milieux géographiques?, Paris, Publications de la Sorbonne.

Baker, Victor, (2009), " Channeled Scabland morphology », in : D. Burr, P.A. Carling et V.R. Baker (Eds), Megaflooding on Earth and Mars, Cambridge, CUP.

Baulig, Henri, (1950), Essais de géomorphologie, Paris, Les Belles Lettres.

Besse, Jean-Marc, (2000), Voir la Terre : six essais sur le paysage et la géographie, Paris, Actes-Sud.

Besse, Jean-Marc, (2009), Le Goût du monde. Exercices de paysage, Paris, Actes-Sud.

Berdoulay, Vincent, (1982), « La métaphore organiciste. Contribution à l'étude du langage des géographes », Annales de Géographie, 91, 507.

Berque, Augustin, (1999), Écoumène. Introduction à l'étude des milieux humains, Paris, Belin.

Bertrand, Georges, (1968), « Paysage et géographie physique globale : esquisse méthodologique », Revue géographique des Pyrénées et du Sud-Ouest, 39, 3.

Birot, Pierre, (1955), Les méthodes de la morphologie, Paris, PUF.

Bishop, Paul, (2007), « Long-term landscape evolution: linking tectonics and surface processes », Earth Surface Processes and Landforms, 32.

Butler, David, (1995), Zoogeomorphology. Animals as geomorphic agents, Cambridge, CUP.

Broc, Numa, (1969), Les montagnes vues par les géographes et les naturalistes de langue française au XVIII siècle, Université de Montpellier (thèse de $3^{\mathrm{e}}$ cycle).

Broc, Numa, (1991), Les montagnes au siècle des Lumières. Perception et représentation, Paris, Éditions du CTHS.

Broc, Numa, Giusti, Christian, (2007), « Autour du Traité de Géographie physique d'Emmanuel de Martonne : du vocabulaire géographique aux théories en géomorphologie », Géomorphologie : relief, processus, environnement, 2.

Brunsden, David, Thornes, John, (1979), « Landscape sensitivity and change », Transactions Institute British Geographers, NS4.

Büdel, Julius, (1977), Klima-Geomorphologie, Berlin, Gebrüder Borntraeger.

Büdel, Julius, (1982), Climatic Geomorphology, Princeton (NJ), PUP.

Burke, Kevin, Gunnell, Yanni, (2008), « The African erosion surface: a continental-scale synthesis of geomorphology, tectonics, and environmental change over the past 180 million years », Geological Society of America Memoir, 201.

Changeux, Jean-Pierre, (2008), Du vrai, du beau, du bien. Une nouvelle approche neuronale, Paris, Odile Jacob.

Chorley, Richard, (1962), " Geomorphology and general systems theory », Geological Survey Professional Paper 500-B, Washington (DC), US Government Printing Office.

Cohen, Claudine, (2004), « Gould et D’Arcy Thompson », C.R. Palevol, 3. 
Corbin, Alain, (1988), Le territoire du vide. L'Occident et le désir de rivage, 1750-1840, Paris, Aubier.

Davis, William Morris, (1899), «The geographical cycle », Geographical Journal, 14, 5. Depraz, Samuel, (2008), Géographie des espaces naturels protégés, Paris, Armand Colin. De Wever, Patrick, (2012), Temps de la Terre, temps de l'Homme, Paris, Albin Michel. Evans, Ian, (2012), « Geomorphometry and landform mapping: What is a landform? », Geomorphology, 137, 1.

Flatrès-Mury, Huguette, (1982), « Analyse et évaluation des paysages », Revue de Géographie de Lyon, 57, 4.

Forrest, Craig, (2010), International Law and the Protection of Cultural Heritage, London, Routledge.

Francioni, Francesco, Lenzerini, Federico, (2008), The 1972 World Heritage Convention. A commentary, Oxford, OUP.

Gell-Mann, Murray, (1994), The Quark and the Jaguar. Adventures in the Simple and in the Complex, New York, W.H. Freeman and Company.

Gilg, Andrew, (1976), « Assessing scenery as a natural resource: causes of variation in Linton's method », Scottish Geographical Magazine, 92, 1.

Giusti, Christian, (2012a), « Sciences du relief ou géomorphologie ? Essai de définition d'un domaine interdisciplinaire entre géosciences et sciences sociales », Cybergeo: European Journal of Geography, URL : http://cybergeo.revues.org/24935

Giusti, Christian, (2012b), Du syndrome de Playfair au paradoxe de Penck. Regards sur les concepts de la géomorphologie, Université de Perpignan (Mémoire HDR).

Giusti, Christian, (2013), « Les Penck en Forêt Noire : une théorie géomorphologique entre ombre et lumière ", Revue de Géographie historique, 2, URL : http://rgh.univ-lorraine.fr/

Giusti, Christian, Calvet, Marc, Gunnell, Yanni, (2013), « Géotope, géofaciès et géosystème : une grille de lecture des paysages géomorphologiques ? ", Collection EDYTEM, $\mathrm{n}^{\circ} 15$.

Goudie, Andrew, Viles, Heather, (2010), Landscapes and geomorphology, Oxford, OUP.

Gould, Stephen Jay, (1989), Wonderful Life. The Burgess Shale and the nature of history, New York, W.W. Norton \& Company.

Gould, Stephen Jay, (1991), La vie est belle : les surprises de l'évolution, Paris, Seuil.

Gould, Stephen Jay, (1994), Un hérisson dans la tempête. Paris, Grasset.

Gould, Stephen Jay, (2002), The Structure of Evolutionary Theory, Cambridge (MA), The Belknap Press of Harvard University Press.

Gould, Stephen Jay, (2006), La structure de la théorie de l'évolution, Paris, Gallimard.

Hack, John, (1960), « Interpretation of erosional topography in humid temperate regions », American Journal of Science, Bradley Volume, 258-A.

Hengl, Tomislav, Reuter, Hannes, (2009), Geomorphometry, Amsterdam, Elsevier.

Hooke, Roy LeB, (1994), « On the efficacy of humans geomorphic agents », GSA Today, 4.

Hooke, Roy LeB, (2000), « On the history of humans as geomorphic agents », Geology, 28. 
Huggett, Richard, (2007), " A history of the systems approach in geomorphology », Géomorphologie : relief, processus, environnement, 2.

Huggett, Richard, Cheesman, Jo, (2002), Topography and the Environment, Harlow, Pearson. Johnson, Douglas, (1940), « Memorandum on the mimeographed outline of the proposed symposium on the geomorphic ideas of Davis and Walther Penck », Annals of the Association of American Geographers, 30, 4.

Jovanović-Kruspel, Stefanie, (2003), The Natural History Museum of Vienna. Where Science meets Art, Wien, Naturhistorisches Museum.

Jovanović-Kruspel, Stefanie, (2012), The Natural History Museum of Vienna. A Guide to the Collections, Wien, Naturhistorisches Museum.

Kennedy, Barbara, (2006), Inventing the Earth: ideas on Landscape development since 1740. Oxford, Blackwell.

Kennel, Sarah, (2013), Charles Marville - Photographer of Paris, Chicago, University of Chicago Press.

Klein, Claude, (1960), « La notion de rythme en morphologie », Norois, 28.

Klein, Claude, (1997), Du polycyclisme à l'acyclisme en géomorphologie. Gap, Éditions Ophrys.

Kuhn, Robert, (1962), The structure of scientific revolutions. Chicago, The University of Chicago Press.

Leopold, Luna, (1969), « Landscape Esthetics. How to quantify the scenics of a river valley », Natural History, 78, 4.

Linton, David, (1968), « The assessment of scenery as a natural resource », Scottish Geographical Magazine, 84, 3.

Luginbühl, Yves, (2012), La mise en scène du monde : la construction du paysage européen, Paris, Éditions du CNRS.

McMillan, Andrew, Powell, John, (1999), « Classification of artificial (man-made) ground and natural superficial deposits. Application to geological maps and datasets in the UK », British Geological Survey Research Reports, RR 99-04.

Migon, Piotr, (2010), Geomorphological Landscapes of the World, Dordrecht, Springer.

Neboit, René, (2010), L'homme et l'érosion, Clermont-Ferrand, PUBP.

Neuray, Georges, (1982), Des paysages. Pour qui ? Pourquoi ? Comment? Gembloux, Presses agronomiques.

Pacione, Michael, (2009), Urban Geography. A global perspective, London, Routledge.

Panizza, Mario, (2001), « Geomorphosites: concepts, methods and example of geomorphological survey », Chinese Science Bulletin, 46, Spl. Bd.

Panizza, Mario, Piacente, Sandra, (2003), Geomorfologia culturale, Bologna, Pitagora Editrice.

Pelletier, Jean, (1982), « Paysage réel et paysage ressenti. Un exemple de paysage à bases physiques : les pays granitiques du pourtour de la Méditerranée », Revue de Géographie de Lyon, 57, 4.

Phillips, Jonathan, (2007), « The perfect landscape », Geomorphology, 84. 
Pica, Alessia, Vergari, Francesca, Fredi, Paola, Del Monte, Maurizio (2014), « The "Aeterna Urbs" geomorphological heritage (Rome, Italy) », Geoheritage (sous presse).

Pitte, Jean-Robert, (1983), Histoire du paysage français, Paris, Tallandier.

Reynard, Emmanuel, (2009), « The problem of scale in geomorphosites studies », in : Christian Giusti, Geomorphosites 2009: Raising the Profile of Geomorphological Heritage through Iconography, Inventory and Promotion. Paris.

Reynard, Emmanuel, Coratza, Paola, Regolini-Bissig, Géraldine, (2009), Geomorphosites, München, Pfeil.

Schumm, Stanley, (1991), To interpret the Earth. Ten ways to be wrong. Cambridge, CUP.

Stallins, Anthony, (2006), « Geomorphology and ecology: Unifying themes for complex systems in biogeomorphology », Geomorphology, 77.

Steffen, Will, Crutzen, Paul, McNeill, John, (2007), « The Anthropocene: are humans now overwhelming the great forces of Nature? », Ambio, 36, 8.

Thompson, D'Arcy Wentworth, (1917), On Growth and Form, Cambridge, CUP.

Thompson, D'Arcy Wentworth, (1942), On Growth and Form, Cambridge, CUP (2 ${ }^{\text {nd }}$ ed.).

Thompson, D’Arcy Wentworth, (1994), Forme et croissance, Paris, Seuil.

Thornes, John, Brunsden, David, (1977), Geomorphology and time, London, Methuen.

Tissier, Jean-Louis, (2003), « Paysage », in : Jacques Lévy et Michel Lussault, Dictionnaire de la géographie et de l'espace des sociétés, Paris, Belin.

Tooth, Stephen, (2009), « Invisible geomorphology? », Earth Surface Processes and Landforms, 34, 5.

Vidal de La Blache, Paul, (1903), Tableau de la géographie de la France. Paris, Hachette.

Viles, Heather, (2013), « Synergistic weathering processes », In: John F. Shroder (ed.) Treatise on Geomorphology, Vol. 4, Ch. 2, San Diego (CA), Academic Press, Elsevier.

Waters, Colin, Zalasiewicz, Jan, Williams, Mark, Ellis, Michael, Snelling, Andrea, (2014), " A stratigraphical basis for the Anthropocene », Geological Society, London, Special Publication, vol. 395.

Wilkinson, Bruce, (2005), « Humans as geologic agents: a deep-time perspective », Geology, 33.

Wohl, Ellen, (2013), « Wilderness is dead: whither critical zone studies and geomorphology in the Anthropocene? », Anthropocene, 2. 
Note ajoutée le 21 octobre 2014

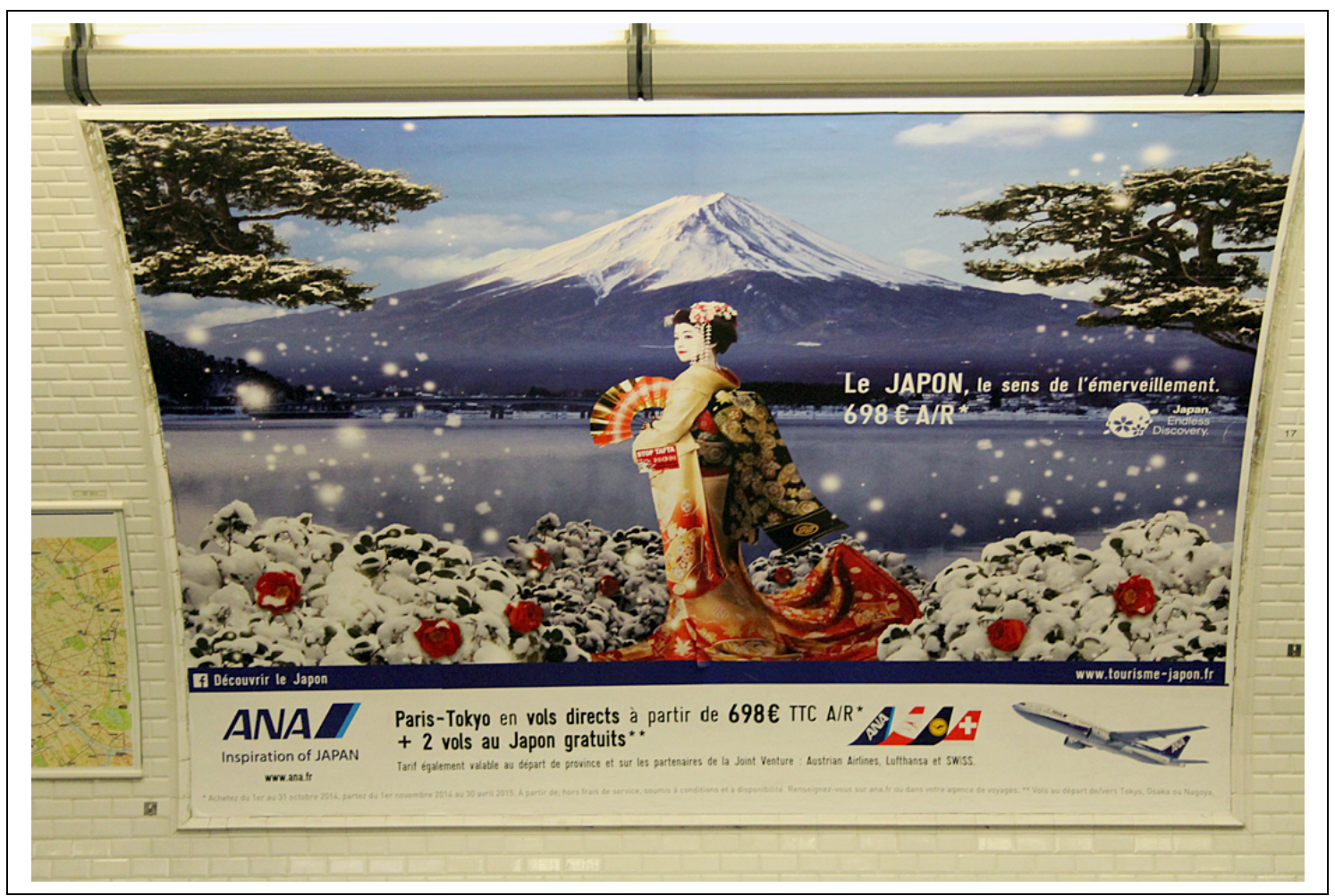

En tant que forme du relief, la valeur iconique du Mont Fuji semble inoxydable : en témoigne cette affiche publicitaire du groupe All Nippon Airways (ANA) et de l'Office National du Tourisme Japonais (JNTO), pour une campagne destinée à promouvoir les attraits culturels et touristiques du Japon.

Photo prise le lundi 20 octobre 2014 à la station « Odéon » de la ligne 4 du métro parisien, par H. KuDLA, étudiante en Master 2 EDTS-GEODEP. 